\title{
Ketoacidosis at diagnosis of type 1 diabetes in children and adolescents from Wielkopolska province in Poland: prevalence, risk factors and clinical presentation
}

\section{ABSTRACT}

Background. Diabetic ketoacidosis (DKA) is a life-threatening condition frequently present at type 1 diabetes diagnosis (T1D). Younger children are at greater risk of developing this acute complication. It is alarming due to worldwide rise in T1D incidence with the greatest increase in children aged $<5$ years. The aim of this study was to identify the prevalence of DKA and factors related to its occurrence in children at T1D diagnosis from Wielkopolska province in Poland. Methods. The study cohort comprised 735 children (girls: 329; boys: 406) aged 0-18 years with new onset T1D admitted to one hospital between 2009 and 2014. The mean age at diagnosis was 9.3 years. DKA was defined as blood $\mathrm{pH}<7.30$. To confirm autoimmune diabetes origin typical autoantibodies were tested.

Results. DKA was diagnosed in $36 \%$ of patients with newly diagnosed T1D. DKA occurred significantly more often in children aged $<4$ years $(p=0.001)$. The highest prevalence of DKA was associated with symp-

Address for correspondence:

Elżbieta Niechciał MD, PhD

Klinika Diabetologii i Otyłości Wieku Rozwojowego

Uniwersytet Medyczny im. Karola Marcinkowskiego w Poznaniu

ul. Szpitalna 27/33, 60-572 Poznań

Phone: +48 618491 420; mobile: +48 606940149

Fax: +48618491492

e-mail: e.niechcial@gmail.com

Clinical Diabetology 2018, 7, 6, 272-278

DOI: $10.5603 /$ DK.2018.0029

Received: 24.09.2018 Accepted: 05.12.2018 toms' duration ( $>28$ days) $(p=0.014)$ and diabetes misdiagnosis ( $p=0.001$ ). Autoantibody against zinc transporter 8 was detected significantly more often in children with DKA ( $p=0.044)$. In the group with DKA, glycated hemoglobin level was significantly higher ( $p=0.0004)$, while insulin and C-peptide levels were lower ( $p=0.0001$ and $p=0.0001$, respectively). Conclusions. The prevalence of DKA is high and its severity is substantial in children with newly diagnosed T1D from Wielkopolska province. Diabetes misdiagnosis, symptoms' duration and age under 4 years are the most common risk factors of DKA development at T1D onset. Autoantibody against zinc transporter 8 is associated with acute T1D onset. (Clin Diabetol 2018; 7, 6: 272-278)

Key words: children, diabetic ketoacidosis, type 1 diabetes onset, autoimmunity, autoantibody against zinc transporter 8

\section{Introduction}

Childhood type 1 diabetes (T1D) is a rising global public health concern, both in developing and economically developed countries. Nowadays, there are 1.94 billion children living all over the world and total 586.000 of children and adolescents below 15 years of age are estimated to have T1D globally. Furthermore, approximately 96.000 new cases of T1D are diagnosed in children aged 0-14 years each year [1]. The overall 
trend across the world is still upwards according to the epidemiological data on the childhood T1D prevalence, with the most pronounced increase in the youngest age group [2-5]. Similarly, in Poland the epidemiological trend is alerted, based on our data from Wielkopolska area, the incidence rate of T1D in children aged 0-14 years increased almost six-fold from $4.2 / 10^{5}$ /year in the early 1970 s to $24.3 / 10^{5} /$ year in 2013 [6]. Witnessed situation has a great impact on children's health since T1D is related not only to long-term complications, but also to short-term consequences, for instance, at diagnosis time. Diabetic ketoacidosis (DKA), one of the most life-threatening severe T1D complications, is highly prevalent in newly diagnosed pediatric patients and its frequency at diagnosis varies from $12.8 \%$ to $80 \%$ depending on a geographic location [7]. Moreover, younger children, aged $<5$ years, are at greater risk of developing DKA [8-11].

On the one hand, the most common rare and primary fatal complication of DKA is a cerebral oedema occurring in $0.3-1 \%$ patients at disease onset, as a result of that DKA is considered as a leading cause of diabetes-related death in children with the mortality rate estimated to be at about $0.15-0.3 \%$ [12-14]. On the other hand, DKA has a negative influence on a residual $\beta$-cell function, therefore, patients with DKA at T1D onset had a lower partial remission incidence in the first six months after diagnosis [15], less a residual $\beta$-cell function and have a worse metabolic control [16]. Those individuals who developed partial remission had significantly lower level of low-density lipoprotein (LDL) 5 years after T1D diagnosis [17].

Commonly, risk factors for DKA in children with newly diagnosed T1D include: young age ( $<5$ years), diagnostic error and delayed treatment, lack of health insurance (USA), lower socioeconomic status, preceding infection, whereas, factors such as first degree relative with T1D, higher parental education and higher background incidence of T1D are consider as a protective factors $[8-10,14]$.

Despite the fact that both the initial symptoms of T1D and the diagnostic criteria are well defined, frequently children are misdiagnosed at the diabetes onset. Particularly, this situation has been observed in infants and toddlers where obtaining the history of classic trio of symptoms, such as polyuria, polydipsia or weight loss, compering to adolescents or young adults is challenging. In young pediatric population frequently the first clinical manifestation is blurred, therefore, the most common diagnostic errors are: respiratory tract disease $(46.3 \%)$, vaginal candidiasis $(16.6 \%)$, gastroenteritis $(16.6 \%)$, urinary tract infection $(11.1 \%)$, gastritis (11.1\%) and appendicitis (3.7\%) [11]. Consequently, due to delayed diagnosis or diagnostic error combined with an inadequate treatment children are more prone to develop severe dehydration and acidosis, which ultimately could lead to cerebral oedema [4].

Regarding the accelerated epidemiological trend of shifting the peaks of age at T1D diagnosis toward younger age groups it seems to be reasonable to evaluate current DKA frequency and investigate factors associated with its presence at the T1D onset in children. Understanding factors which are associated with DKA occurrence at diagnosis may help to decrease the number of children presenting with severe DKA, thereby, it could contribute to reducing acute complications and the risk of death at T1D onset.

The purpose of this study was to identify the prevalence of DKA and factors related to its occurrence in children and adolescents at T1D diagnosis from Wielkopolska province, Poland.

\section{Material and methods Data collection}

The study cohort comprised 735 European Caucasian children (girls: 329; boys: 406) aged 0-18 years with newly diagnosed T1D admitted to Department of Pediatric Diabetes and Obesity, Poznan University of Medical Sciences in a six-year period between January $1^{\text {st }}, 2009$ and December 315t, 2014. The study was approved by the Poznan University of Medical Sciences Ethics Committee (decisions No 960/12). Informed consent was obtained from all individual participants included in the study.

\section{Diabetes diagnosis}

Diabetes was diagnosed based upon WHO criteria, including fasting plasma glucose $\geq 126 \mathrm{mg} / \mathrm{dl}$ (7.0 mmol/l), 2-h postprandial plasma glucose $\geq 200 \mathrm{mg} / \mathrm{dl}$ (11.1 mmol/l), during an oral glucose tolerance test, classic symptoms of hyperglycemia or hyperglycemic crisis, as well as random plasma glucose concentration of $\geq 200 \mathrm{mg} / \mathrm{dl}(11.1 \mathrm{mmol} / \mathrm{l})$. The first day of insulin administration was at T1D diagnosis.

\section{Clinical and laboratory data}

Standard laboratory tests, including blood glucose, capillary blood gases and blood or urinary ketones were performed in every patient. DKA was defined as blood glucose concentration $>250 \mathrm{mg} / \mathrm{dl}(13.9 \mathrm{mmol} / \mathrm{l})$, blood $\mathrm{pH}<7.30$, concentration of $\mathrm{HCO} 3<15 \mathrm{mmol} / \mathrm{l}$, detection of ketones in the urine or elevated ketones in the serum, anion gap $>12$. It was considered as mild, moderate and severe if $\mathrm{pH}$ was $<7.3 \geq 7.2,<7.2$ $\geq 7.1$ and $<7.1$, respectively. According to the obtained results, children were divided into two groups: non DKA 
group and DKA group. Fasting C-peptide and insulin levels were evaluated as a measurement of residual $\beta$-cell function at T1D onset. The blood samples were collected before an initial insulin therapy and assayed by the clinical laboratory immediately after the samples were obtained. C-peptide concentration was analysed using radioimmunoassay (C-PEP II-RIA-CT, DIAsource Immunoassay, S.A, Louvain-la-Neuve, Belgium), with the normal range: $0.59-1.54 \mathrm{pmol} / \mathrm{ml}$, while insulin level was assessed by Chemiluminescent Microparticle Immunoassay (The Insulin ARCHITECT System, Abbott Laboratories, Illinois, USA), with the fasting normal range $<15 \mathrm{uU} / \mathrm{ml}$. Glycated haemoglobin $\left(\mathrm{HbA}_{1 \mathrm{c}}\right)$ was measured using NGSP-certified method $\left(\mathrm{HbA}_{1 \mathrm{c}} \mathrm{ARCHI}-\right.$ TECT System, Abbott Laboratories, Illinois, USA) with non-diabetic values $<5.7 \%$. To confirm autoimmune diabetes origin typical autoantibodies were tested. Autoantibodies against glutamic acid decarboxylase (GADA) and antibodies to tyrosine phosphatase-like antigen (IA-2) were measured by ELISA kits. The normal reference range for GAD-ab and IA2-ab was $\leq 1$ $\mathrm{U} / \mathrm{mL}$ (Anti-Glutamic Acid Decarboxylase (GAD) and Anti-Tyrosine Phosphatase (IA2) (RIA EUROIMMUN, Lubeck, Germany). Insulin autoantibodies (IAA) were measured by immunoassays with the normal reference range $<5.5 \%$ (AIA-100, DIAsource Immunoassay, S.A, Louvain-la-Neuve, Belgium). Additionally, 219 patients were tested to autoantibodies to zinc transporter-8 (ZnT8-ab) level using enzyme-linked immunosorbent assay. The upper limit of the normal range was $15 \mathrm{U} / \mathrm{mL}$ (Elisa-ZnT8 AbTM, RSR, Cardiff, United Kingdom).

A questionnaire on diabetes from first symptom(s) to diagnosis was completed by children's caregivers. Factors affecting the risk of DKA development such as age, sex, residence (urban vs. rural), symptoms duration, initial diagnosis, socioeconomic status, parental education, first degree relative with T1D were investigated in all study population. The lack of health insurance was not considered as a risk factor, because insured and uninsured children have the same access to the medical care in Poland.

The data was divided into four age groups: 0-4, 5-9, 10-14 and 14-18 years. Four patients were excluded from our study: two with neonatal diabetes, one with MODY 2 and one with T2D.

\section{Statistical methods}

All data are expressed as mean standard deviations ( \pm SD), unless otherwise stated. The analysed data came from the interval and nominal scale. Student's $t$-test was used to compare the two groups, and the Mann-Whitney test was applied in case of data does not follow a normal distribution. The assumption of
Table 1. Clinical features of children at T1D diagnosis

\begin{tabular}{lc}
\hline Variable & $\mathbf{n}=\mathbf{7 3 5}$ \\
\hline Sex $(\mathrm{M}: \mathrm{F})$ & $329: 406$ \\
Age $(\mathrm{yr})$ & $9.3 \pm 4.5$ \\
Blood glucose [mg/dl] & $432 \pm 196$ \\
Blood ketones [mmol/L] & $3.5 \pm 1.9$ \\
$\mathrm{pH}$ & $7.35 \pm 0.15$ \\
$\mathrm{HbA}$ & $11.3 \pm 2.0$ \\
Insulin [uU/ml] & $2.5 \pm 1.7$ \\
C-peptide [pmol/ml] & $0.4 \pm 0.24$ \\
DKA overall, \% (n) & $36(265)$ \\
Mild DKA overall, \% (n) & $12.9(95)$ \\
Moderate DKA overall, \% (n) & $14.5(107)$ \\
Severe DKA overall, \% (n) & $8.6(63)$ \\
\hline
\end{tabular}

$\mathrm{HbA}_{1 c}$ - glycated haemoglobin; DKA — diabetic ketoacidosis

normality was checked by Kolmogorov-Smirnov test. Nominal data were analysed with the $\chi^{2}$ test or Fisher's exact test. When more than two groups were compared simultaneously, univariate analysis of variance with the Tukey post-hoc test was performed. All tests were analysed at the significance level of $\alpha=0.05$. Statistical analysis was performed using Statistica 10.0 software (Stat Soft Inc, Tulsa, USA).

\section{Results}

During six years of our study we identified 735 new cases of T1D in children aged 0-18 years from Wielkoposka province. The frequency of T1D diagnosis among both genders was comparable and it was reported in 329 girls (44.7\%) and 406 boys (55.3\%). The mean age at diagnosis was 9.3 years (SD \pm 4.5 ). Laboratory results are shown in Table 1.

DKA was diagnosed in $36 \%$ of patients with newly diagnosed T1D ( $n=267)$, of whom 12.9\% ( $n=95)$ developed mild, $14.5 \%$ moderate and $8.6 \%$ severe (Table 1). There was no difference in the frequency of DKA between girls and boys (49.3\% vs. $50.7 \%)$. When comparing studied age groups it was noted that the occurrence of DKA was higher in younger individuals. In children aged 0-4, 5-9, 10-14 and 15-18 years DKA was present in 82 (48.5\%), 90 (34.7\%), 72 (31.4\%) and $22(28.2 \%)$ cases, respectively. In the youngest group aged $<4$ years it occurred significantly more often ( $p=0.001$ ). Comparable severity of DKA was observed in all age-groups. Mostly mild or moderate DKA was diagnosed (Figures 1, 2).

Blood ketones, blood glucose and $\mathrm{HbA}_{1 \mathrm{c}}$ levels were significantly higher in children with DKA compared to children without it at diagnosis time ( $p=0.0001, p=0.00001, p=0.0004$, respec- 


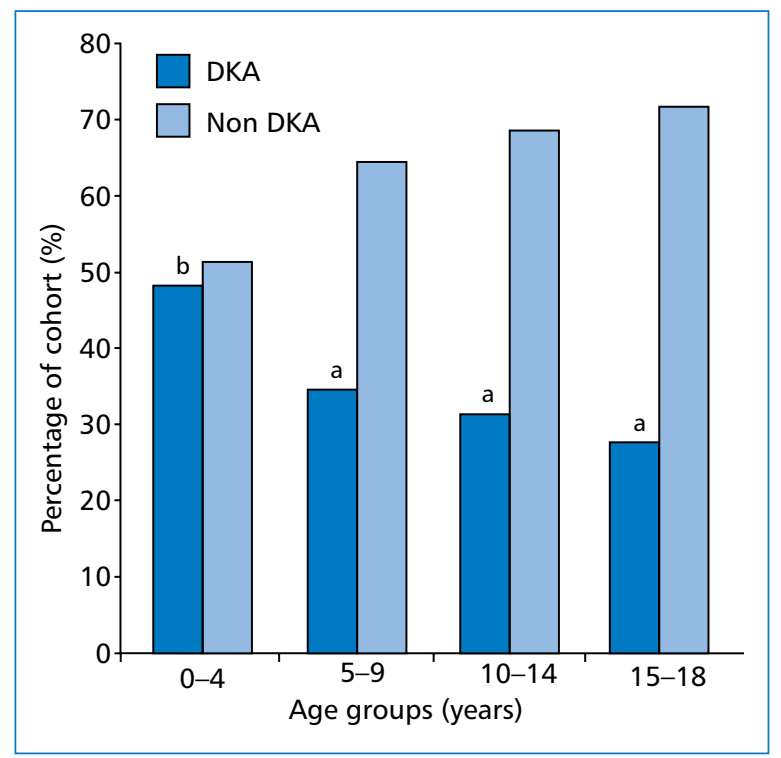

Figure 1. DKA and non DKA ratio in children with newly diagnosed T1D by age. a, $b$ - groups followed by the same letter do not differ statistically significantly

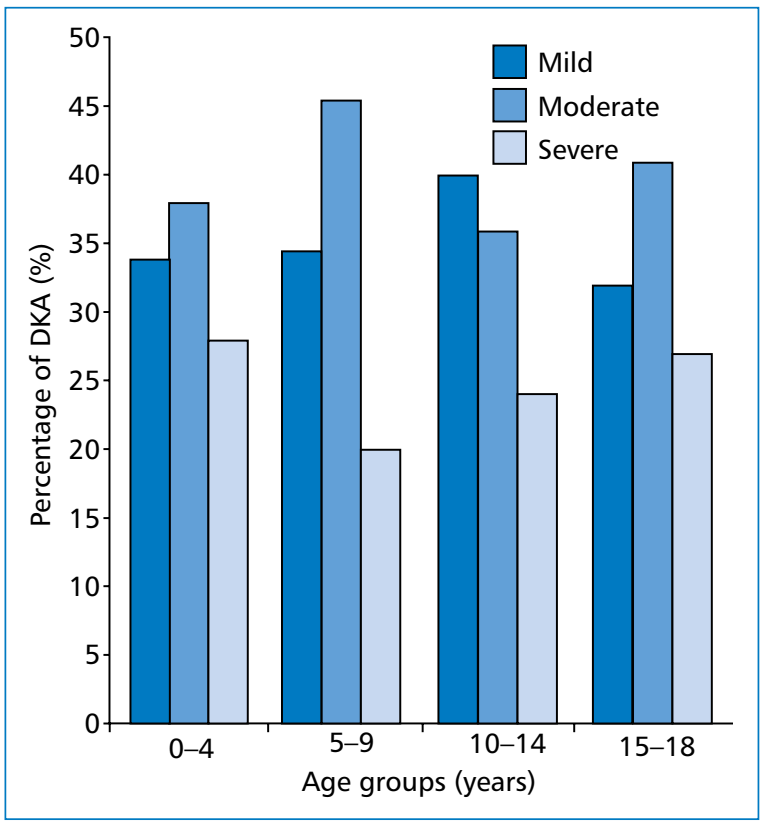

Figure 2. Distribution of DKA degree by age at T1D onset

tively), while insulin and C-peptide levels were lower $(p=0.0001$ and $p=0.0001$, respectively) (Table 2).

Two major risk factors responsible for a greater prevalence of DKA were reported in children with newly diagnosed T1D. The highest DKA frequency was associated with longer symptoms' duration ( $>28$ days) $(p=0.014)$ and initiall diabetes misdiagnosis $(p=$ 0.001 ) (Table 2). While, factors such as rural or urban residence, sex, pre-hospitalisation, parental education, lower socioeconomic status or first degree relative with T1D did not affect DKA occurrence in our study.

At T1D onset the spectrum of antibodies and their correlations to DKA presence were investigated. ZnT8-ab was detected significantly more often in children with DKA compared to children without DKA ( $p=$ $=0.044$ ). Moreover, significantly higher titers of ZnT8-ab [448.0 (25.7-524.5) vs. 284.3 (49.5-524.5) U/ml, $\mathrm{p}<0.039$ ] were found among individuals with DKA. There was no relationship between DKA presence or its severity and other typical autoantibodies (Table 3).

\section{Discussion}

Undeniably, the frequency of DKA at the T1D onset in Poland is unacceptably high. This is reflected not only in our study, which showed that $36 \%$ of children presented DKA at the diagnosis time, but also other reports from Poland confirmed our findings [18, 19]. There are many possible reasons responsible for observed situation.

First of all, recently the dynamic changes in childhood T1D prevalence has been observed in Poland. The incidence of T1D has increased almost six-fold since the first epidemiological studies conducted in the early 1970s in Wielkopolska province, with the current incidence rate estimated at around $24.3 / 10^{5} /$ year in children under 15 years [6]. Likewise, the growing number of children with newly diagnosed T1D was reported in the other regions of Poland [20-22]. Despite the fact that T1D is one of the most common chronic diseases occurring in pediatric population it was shown that physicians-related factor, such as misdiagnosis at the first medical consultation, may play a key role in DKA development in children at T1D onset. Diagnostic error was previously reported as a factor having a negative impact on DKA occurrence. Several studies showed that children not recognised to have T1D onset at first medical visits had threefold increased risk of presenting in DKA. This risk was independent of the presence or absence of infection preceding diagnosis, however, misdiagnosis occurred more frequently in younger children [8]. Similarly, this study demonstrated that the delayed diagnosis and symptoms' duration longer than 28 days are positively correlated with the higher occurrence of DKA in children at disease onset. Our findings suggest that many primary care physicians and pediatricians may have difficulties in recognising this condition or are not aware of the possibility of diabetes diagnosis in children, as a consequence, it contributes to treatment delay. Probably, health providers are unfamiliar with T1D due to previously reported low T1D incidence rate among Polish children, therefore, T1D might be 
Table 2. Clinical features of children at T1D onset with and without DKA

\begin{tabular}{lccc}
\hline Variable & Children without DKA $(\mathbf{n}=\mathbf{4 7 0})$ & Children with DKA $(\mathbf{n}=\mathbf{2 6 5})$ & P-value \\
\hline Blood glucose $[\mathrm{mg} / \mathrm{dl}]$ & $407 \pm 178$ & $474 \pm 218$ & 0.00001 \\
Blood ketones $[\mathrm{mmol} / \mathrm{L}]$ & $2.3 \pm 1.7$ & $4.8 \pm 1.3$ & 0.0001 \\
$\mathrm{HbA}_{1 \mathrm{c}}(\%)$ & $11.1 \pm 2.2$ & $11.6 \pm 1.8$ & 0.0004 \\
Insulin [uU/mL] & $2.8 \pm 1.8$ & $1.8 \pm 1.4$ & 0.0001 \\
C-peptide [pmol/ml] & $0.57 \pm 2.3$ & $0.3 \pm 0.16$ & 0.0001 \\
Symptoms' duration (days) & $<28$ & $>28$ & 0.014 \\
Diabetes misdiagnosis* & GP visits $<3$ & GP visits $>3$ & 0.001 \\
\hline
\end{tabular}

*Number of GP visits. NS — non-significant; GP — general practitioner

Table 3. Autoimmune markers of patients with and without DKA at T1D onset

\begin{tabular}{|c|c|c|c|}
\hline Variable & Children with DKA $(n=265)$ & Children without DKA $(n=470)$ & P-value \\
\hline \multicolumn{4}{|l|}{ Autoantibodies } \\
\hline Positive GAD-ab, \% (n) & $70.0(186)$ & $72.0(338)$ & NS \\
\hline GAD-ab titre [U/ml], (IQR) & $3.2(0.7-18.6)$ & $3.8(0.7-17.7)$ & NS \\
\hline Positive IAA, \% (n) & $52.0(138)$ & $51.0(240)$ & NS \\
\hline IAA titre [U/ml] & $5.6(4.8-7.5)$ & $5.5(4.8-7.2)$ & NS \\
\hline Positive IA2-ab, \% (n) & $79.0(209)$ & $78.0(368)$ & NS \\
\hline IA2-ab titre [U/ml] & $6.2(1.4-18.9)$ & $7.4(1.3-20.5)$ & NS \\
\hline Positive ZnT8-ab, \% (n) & $93.0(138)$ & $79.0(55)$ & 0.044 \\
\hline ZnT8-ab titre [U/ml] & $448.0(25.7-524.5)$ & $284.3(49.5-524.5)$ & 0.039 \\
\hline \multicolumn{4}{|l|}{ Combination of positive autoantibodies } \\
\hline IAA + GAD-ab, \% (n) & $6.4(17)$ & $6.2(29)$ & NS \\
\hline IAA + IA2-ab, \% (n) & $7.2(19)$ & $8.1(38)$ & NS \\
\hline GAD-ab + IA2-ab, \% (n) & $25.0(67)$ & $24.8(117)$ & NS \\
\hline IAA + ZnT8, \% (n) & $0.3(1)$ & $0.0(0)$ & NS \\
\hline IA2-ab + ZnT8, \% (n) & $25.2(67)$ & $27.5(129)$ & NS \\
\hline GAD-ab +ZnT8, \% (n) & $24.7(65)$ & $24.5(116)$ & NS \\
\hline IAA + GAD-ab + IA2-ab, \% (n) & $22.6(60)$ & $23.2(109)$ & NS \\
\hline IAA + GAD-ab + ZnT8, \% (n) & $4.5(12)$ & $4.4(21)$ & NS \\
\hline$I A A+I A 2-a b+Z n T 8, \%(n)$ & $14.3(45)$ & $16.6(83)$ & NS \\
\hline GAD-ab + IA2-ab + ZnT8, \% (n) & $22.6(60)$ & $21.0(98)$ & NS \\
\hline GAD-ab +IAA + IA2-ab + ZnT8, \% (n) & $39.2(104)$ & $39.6(186)$ & NS \\
\hline
\end{tabular}

Data are expressed as medians; unless otherwise stated. IQR — interquartile range; NS — non-significant

still consider as a rare disease in pediatric population. As a result of that, not only children living in countries with low prevalence of T1D are at risk of developing of DKA at disease onset, but also those living in countries with rapid changes in T1D incidence.

Secondly, age-related differences in the frequency of DKA at T1D onset was observed in this study, subsequently, almost half of children aged 0-4 developed DKA, as opposed to older individuals who had a lower prevalence of DKA. Comparable high frequency of DKA in younger children with new-onset diabetes was reported from other studies worldwide [7, 9, 10, 12]. It seems to be important, because common signs of T1D often resemble symptoms of other disease. Particularly, young children could present asymptomatic manifestation of disease, therefore, they may be misdiagnosed as having pneumonia, reactive airways disease (asthma) or bronchiolitis [11]. Consequently, those children receive an inappropriate treatment, such as glucocorticoids and/or sympathomimetic drugs, which aggravate the metabolic disorder. Again, physicians should be aware of the possibility of diabetes occurrence in young children, particularly when the peak incidence is still shifting to a younger age $[1,2,4,5]$. On the one side, concomitant illness, especially infections, may cause an increased insulin requirement and, thereby may 
provoke a faster decompensation of already affected by the destructive process $\beta$-cell function leading to accelerated disease manifestation. As a result of that, it is reasonable to put more effort on medical education, because primary care physicians may help in reducing number of children presenting in DKA.

On the other side, occurrence of DKA is not only associated with acute complications at disease onset, but also it might influence later clinical course of T1D, particularly in terms of entering a remission phase. There are several clinical and metabolic factors interfering the presence and length of the remission period, which depends partly on the recovery of $\beta$-cell function [23]. Neylon et al. demonstrated that children who experienced moderate or severe DKA at T1D diagnosis are less likely to develop a partial remission phase and their honeymoon period is shorter compared to those without DKA at disease onset [24]. Moreover, younger age at diagnosis is related to the worst preserved $\beta$-cell function, consequently, children under 2 years of age had a negligible chance to develop honeymoon phase and even if, the duration of this stage was shorter than in children over 5 years of age $[15,23]$. Therefore, thus indicates that the higher frequency of DKA and younger age at diagnosis could be associated with more aggressive $\beta$-cell destruction process and less residual insulin secretion at the time of diagnosis. Similarly, we reported that children with DKA had greater decline of $\beta$-cell function compared to individuals without DKA at T1D onset. Then, residual insulin secretion may have a negative impact on partial remission incidence or glycaemia control in those with DKA, nevertheless, the limitation of this study is the lack of stimulated C-peptide levels measurement and further follow-up.

Regarding autoimmune markers, we investigated correlation between typical T1D autoantibodies and DKA presence in children at the disease onset. Interestingly, higher occurrence and titers of ZnT8-ab were reported more frequently in those who were diagnosed with DKA. We did not find any associations between other autoimmune markers and DKA diagnosis. Relationship between presence of ZnT8-ab and severity of diabetes clinical manifestation is still ambiguous. However, ZnT8-ab is highly $\beta$-cells specific compared to other autoimmune markers. ZnT8 is an autoantigen, exclusively expressed in insulin containing secretory granules of pancreatic $\beta$-cells [25]. Our findings might suggest that Znt8-ab is associated with a strong autoimmune attack and more aggressive autoimmune damage of $\beta$-cell population.

Finally, it seems plausible that the correlation between insulin resistance and T1D clinical manifestation exists. Assuming that insulin resistance increases with aging and the normal growth, especially during puberty period, young adults and teenager are more insulin resistant compared to younger children. Consequently, at T1D onset destructive process is not yet advanced and there are still existing $\beta$-cells with preserved function. In such a situation older children may present T1D initial symptoms earlier and slower, which may prevent DKA development at T1D diagnosis. This hypothesis seems to be applicable due to increasing number of overweight and obese children with unclear diabetes diagnosis [26, 27]. In contrast, younger children are characterized by a high insulin sensitivity which may delay T1D symptoms occurrence to the moment when $\beta$-cells destruction has reached their critical deficit. Perhaps, it explains highest DKA prevalence at T1D diagnosis in young children.

To conclude, the frequency of DKA in newly diagnosed with T1D children was significant in this region of Poland. More than one-third of T1D children were presented with DKA at the diagnosis time. There was correlation between diabetes misdiagnosis, symptoms' duration, age under 4 years and presentation at DKA state. Severity of DKA was particularly substantial in the youngest children. ZnT8-ab is associated with the worst general condition at T1D onset. In the face of increasing incidence of T1D, especially in the youngest children, the pediatricians and general practitioners require further education about diabetes presentation in children of different age and possible diagnostic errors.

\section{Study limitations}

Some limitations should be taken into account when interpreting the results of this study. ZnT8-ab assessment was not performed in all study population, consequently, the analysis might not reflect exact clinical meaning of these autoantibodies and their role in autoimmune process. Another weakness of this study was carrying out the analysis only among children and adolescents from Wielkopolska province, then, findings may not represent DKA prevalence at T1D onset in all polish pediatric population. Moreover, the effect of DKA on partial remission was not investigated in this study, because we did not evaluate stimulated C-peptide levels and we not followed-up study participants. Finally, some additional potential factors influencing DKA prevalence were not assessed in this study such as family structure, time of the year, pattern and frequency of symptoms, preceding infections or febrile illnesses, which have previously been found to affect DKA occurrence in some although not all studies in children and adolescents.

\section{Acknowledgments}

Parts of this study were presented in abstract form at the 52nd Annual Meeting of the European Asso- 
ciations for the Study of Diabetes, Munich, Germany, 13-16 September 2016.

This research did not receive any specific grant from funding agencies in the public, commercial, or not-for-profit sectors.

\section{Statement of competing interests}

The authors have no conflicts of interest to disclose.

\section{Authors" contributions}

EN, BS, MM, PF contributed substantially to the conception and design of the study. EN, BS, PF were responsible for data collection and/or analysis, while MM performed statistical analysis. All authors took a part in writing or revising the manuscript and approving the final version of the manuscript to be published. EN is the guarantor of this work and, as such, had full access to all the data in the study and take responsibility for the integrity of the data and the accuracy of the data analysis.

\section{REFERENCES}

1. Aldworth J, Chris Pa, Jacobs E. IDF Diabetes Atlas Eighth Edition; 2017.

2. Menke A, Orchard TJ, Imperatore G, et al. The prevalence of type 1 diabetes in the United States. Epidemiology. 2013; 24(5): 773-774, doi: 10.1097/EDE.0b013e31829ef01a, indexed in Pubmed: 23903880

3. Dabelea D, Mayer-Davis EJ, Saydah S, et al. SEARCH for Diabetes in Youth Study. Prevalence of type 1 and type 2 diabetes among children and adolescents from 2001 to 2009. JAMA. 2014; 311(17): 1778-1786, doi: 10.1001/jama.2014.3201, indexed in Pubmed: 24794371

4. Soltesz G, Patterson CC, Dahlquist G. Worldwide childhood type 1 diabetes incidence - What can we learn from epidemiology? Pediatric Diabetes. 2007; 8: 6, doi: 10.1111/j.13995448.2007.00324.x

5. Stanescu DE, Lord K, Lipman TH. The epidemiology of type 1 diabetes in children. Endocrinol Metab Clin North Am. 2012; 41(4): 679-694, doi: 10.1016/j.ecl.2012.08.001, indexed in Pubmed: 23099264

6. Rewers M, Stone RA, LaPorte RE, et al. Poisson regression modeling of temporal variation in incidence of childhood insulindependent diabetes mellitus in Allegheny County, Pennsylvania, and Wielkopolska, Poland, 1970-1985. Am J Epidemiol. 1989; 129(3): 569-581, indexed in Pubmed: 2916550.

7. Usher-Smith JA, Thompson M, Ercole A, et al. Variation between countries in the frequency of diabetic ketoacidosis at first presentation of type 1 diabetes in children: a systematic review. Diabetologia. 2012; 55(11): 2878-2894, doi: 10.1007/s00125012-2690-2, indexed in Pubmed: 22933123.

8. Usher-Smith JA, Thompson MJ, Sharp SJ, et al. Factors associated with the presence of diabetic ketoacidosis at diagnosis of diabetes in children and young adults: a systematic review. BMJ. 2011; 343(jul07 1): d4092-d4092, doi: 10.1136/bmj.d4092.

9. Lee HJ, Yu HW, Jung HW, et al. Factors Associated with the Presence and Severity of Diabetic Ketoacidosis at Diagnosis of Type 1 Diabetes in Korean Children and Adolescents. J Korean Med Sci. 2017; 32(2): 303-309, doi: 10.3346/jkms.2017.32.2.303, indexed in Pubmed: 28049242.

10. Onyiriuka AN, Ifebi E. Ketoacidosis at diagnosis of type 1 diabetes in children and adolescents: frequency and clinical characteristics.
J Diabetes Metab Disord. 2013; 12(1): 47, doi: 10.1186/22516581-12-47, indexed in Pubmed: 24355514.

11. Pawłowicz $M$, Birkholz D, Niedźwiecki $M$ et al. Difficulties or mistakes in diagnosing type 1 diabetes in children? - demographic factors influencing delayed diagnosis. Pediatr Diabetes. 2009;10(8):542-549. doi: 10.1111/j.1399-5448.2009.00516.x.

12. Neu A, Willasch A, Ehehalt S, et al. DIARY Group Baden-Wuerttemberg. Ketoacidosis at onset of type 1 diabetes mellitus in children - frequency and clinical presentation. Pediatr Diabetes. 2003; 4(2): 77-81, indexed in Pubmed: 14655263.

13. Piva J, Czepielewski M, Garcia P, et al. Current perspectives for treating children with diabetic ketoacidosis. Jornal de Pediatria. 2007; 0(0), doi: 10.2223/jped.1707.

14. Wolfsdorf J, Glaser N, Agus M, et al. ISPAD Clinical Practice Consensus Guidelines 2018: Diabetic ketoacidosis and the hyperglycemic hyperosmolar state. Pediatric Diabetes. 2018; 19: 155-177, doi: 10.1111/pedi.12701.

15. Abdul-Rasoul M, Habib H, Al-Khouly M. 'The honeymoon phase' in children with type 1 diabetes mellitus: frequency, duration, and influential factors. Pediatr Diabetes. 2006; 7(2): 101-107, doi: 10.1111/j.1399-543X.2006.00155.x, indexed in Pubmed: $1662971 \underline{6}$.

16. Fredheim S, Johannesen J, Johansen A, et al. Danish Society for Diabetes in Childhood and Adolescence. Diabetic ketoacidosis at the onset of type 1 diabetes is associated with future $\mathrm{HbA}_{1 \mathrm{c}}$ levels. Diabetologia. 2013; 56(5): 995-1003, doi: 10.1007/s00125-0132850-z, indexed in Pubmed: 23389397.

17. Nwosu BU, Zhang Bo, Ayyoub SS, et al. Children with type 1 diabetes who experienced a honeymoon phase had significantly lower LDL cholesterol 5 years after diagnosis. PLoS One. 2018; 13(5): e0196912, doi: 10.1371/journal.pone.0196912, indexed in Pubmed: 29768449.

18. Olak-Białoń $B$, Deja $G$, Jarosz-Chobot $P$, et al. The occurrence and analysis of chosen risk factors of DKA among children with new onset of DMT1. Wieku Rozw. 2007.

19. Szypowska A, Ramotowska A, Grzechnik-Gryziak M, et al. High Frequency of Diabetic Ketoacidosis in Children with Newly Diagnosed Type 1 Diabetes. Journal of Diabetes Research. 2016; 2016 : 1-5, doi: 10.1155/2016/9582793.

20. Jarosz-Chobot P, Polanska J, Szadkowska A, et al. Rapid increase in the incidence of type 1 diabetes in Polish children from 1989 to 2004, and predictions for 2010 to 2025. Diabetologia. 2010; 54(3): 508-515, doi: 10.1007/s00125-010-1993-4.

21. Jarosz-Chobot P, Deja G, Polanska J. Epidemiology of type 1 diabetes among Silesian children aged 0-14 years, 1989-2005. Acta Diabetol. 2010; 47(1): 29-33, doi: 10.1007/s00592-009-0094-7, indexed in Pubmed: 19183839.

22. Szalecki M, Wysocka-Mincewicz M, Ramotowska A, et al. Epidemiology of type 1 diabetes in Polish children: A multicentre cohort study. Diabetes Metab Res Rev. 2018. doi: 10.1002/dmrr.2962.

23. Lombardo F, Valenzise M, Wasniewska M, et al. Two-year prospective evaluation of the factors affecting honeymoon frequency and duration in children with insulin dependent diabetes mellitus: the key-role of age at diagnosis. Diabetes Nutr Metab. 2002; 15(4): 246-251, indexed in Pubmed: 12416662.

24. Neylon OM, White M, O ' Connell MA, et al. Insulin-dose-adjusted $\mathrm{HbA1c}$-defined partial remission phase in a paediatric population — when is the honeymoon over? Diabetic Medicine. 2013; 30(5): 627-628, doi: 10.1111/dme.12097.

25. Wenzlau JM, Juhl K, Yu L, et al. The cation efflux transporter ZnT8 (Slc30A8) is a major autoantigen in human type 1 diabetes. Proc Natl Acad Sci U S A. 2007; 104(43): 17040-17045, doi: 10.1073/ /pnas.0705894104, indexed in Pubmed: 17942684.

26. Cizza G, Brown RJ, Rother KI. Rising incidence and challenges of childhood diabetes. A mini review. J Endocrinol Invest. 2012; 35(5): 541-546, doi: 10.3275/8411, indexed in Pubmed: 22572768.

27. Gertig AM, Niechciał E, Skowrońska B, et al. New-Onset Diabetes in Obese Adolescents ? Type 1 or Type 2 Diabetes? Comparative Cases Report. J Diabetes Metab. 2012:1-5. doi: 10.4172/21556156.S11-006. 